\title{
Microstructured ceramic and metallic implant surfaces and their impact on the viscosity of a synovia fluid substitute
}

\begin{abstract}
This study evaluates the effect of ring-shaped microstructures on the rheological properties of a synovial fluid substitute. Two different materials that are frequently used in endoprostheses have been chosen in order to study the lubricating effect of femtosecond-laser microstructured implant surfaces by measuring the apparent dynamic viscosity of the lubrication fluid. The two different materials are the ceramic $\mathrm{Al} 2 \mathrm{O} 3$ and the metal alloy CoCrMo. The results show that an increase in the viscosity of the synovia fluid substitute can be achieved by specific microstructuring. An increase of viscosity of up to $40 \%$ compared to an unstructured reference was observed with ring-shaped microstructures with a diameter of $100 \mu \mathrm{m}$, a texture area density of $2.5 \%$ and an aspect ratio of 0.66 . The measurements have also shown that the ceramic material resulted in slightly higher viscosity values, compared to the metal alloy which can explained by dimensional deviations of the microstructures caused by the laser microstructuring.
\end{abstract}

Keywords: Surface modification, rheology, joint implants

https://doi.org/10.1515/cdbme-2020-3158

\section{Introduction}

The demand for better endoprostheses has encouraged many studies that try to understand the underlying tribological and lubrication mechanisms of articulating joints as well as improving the longevity of endoprostheses [2, 8, 9, 12]. Many approaches such as better materials or material modifications like microstructuring were investigated [1,13]. Research has already been carried out on the effect of different microstructures in endoprostheses [2,3]. Results have shown that micro-pockets lead to a significant improvement in the lubricant film thickness compared to untreated surfaces [7,
10]. In order to improve joint lubrication, the surfaces of the articulating components can be modified by pulsed femtosecond-laser microstructuring. Femtosecond-lasers are an ideal tool for such microstructures, due to their accuracy. The microstructuring of the implant surface can lead to an increase of the viscosity of the synovial fluid between the articulating joint surfaces and therefore have a positive effect on lubrication [4].

The goal of this approach is to understand and improve hydrodynamic lubrication of joint prostheses by introducing microstructures and therefore lower particle abrasion through wear. Joints exhibit complex and transient load as well as kinematic multi-axis motions that operate in a mixed, elastohydrodynamic lubrication regime [6]. Despite extensive research, these mechanisms are still not fully understood. Especially the wear of the surfaces in endoprostheses remains a major problem [7]. In order to better understand such lubrication mechanisms, an investigation on the impact of microstructures on the viscosity of the non-Newtonian lubricating fluid, called synovia fluid, is carried out. The synovia fluid between the articulating joints is produced by the surrounding tissue of the joint [5] and plays a vital role in the longevity of a joint replacement [11].

In a previous study, it was shown that the use of various microstructures has an improving effect on the apparent viscosity of the synovial substitute [4]. The aim of this study is to investigate the effect of different medical grade materials and microstructures on the rheological properties of a synovia fluid substitute. The pulsed laser structuring process of different materials can lead to dimensional deviations of the microstructures, which may result in different rheological properties. Besides the most used soft-hard combination of joint replacements, hard-hard combinations are still relevant to this day [13]. In order to improve such hard-hard combinations, the microstructuring of medical grade metal and ceramic components were carried out.

\section{Materials and methods}

The disks for the laser structuring and the subsequent viscosity measurements have a diameter of $25 \mathrm{~mm}$ and a height of $6 \mathrm{~mm}$. 
The metal disks are made of a medical CoCrMo alloy type Ergiloy 9.9135HL from the company Zapp Precision Metals $\mathrm{GmbH}$ (Ratingen, Germany) and the ceramic discs are made of a zirconiumoxide-enriched aluminumoxide ELEC PLUS $\AA$ from the company HiPer Ceramics $\mathrm{GmbH}$ (Oberkrämer, Germany). Dimensions of the structuring area on the samples are given in Error! Reference source not found., with d as the microstructure's diameter, $t$ as the microstructure depth, $w$ for the microstructure distance and a for the microstructure's width.

Microstructuring was carried out with a femtosecond fiber laser of the type TruMicro 5050 Femto Edition (Trumpf GmbH \& Co. KG, Ditzingen, Germany) and a pulse duration of $800 \mathrm{fs}$. A ring area of the disks with an outer radius of 12.5 $\mathrm{mm}$ and an inner radius of $5 \mathrm{~mm}$ was microstructured in order to reduce the shear rate reducing effect. Figure 1 shows how the structuring of the disks are to be carried out.

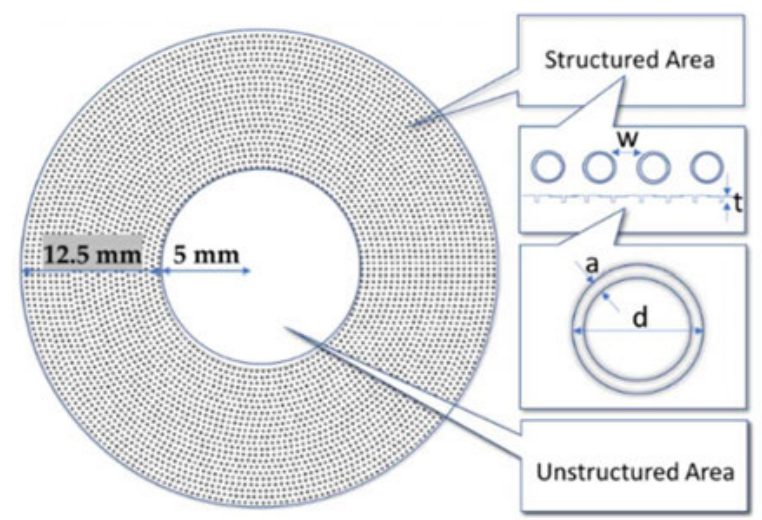

Fig. 1: Schematic depiction of microstructures on the disk samples.

The rheometer MCR 702 (Anton Paar Germany GmbH, Ostfildern, Germany) with its software RheoCompass (Version 1.20.493) was used for the parallel plate rheological measurements. The measurement of the apparent dynamic viscosity $\eta$ ' were carried out with a reduced gap of $0.1 \mathrm{~mm}$ using a modified rheometer setup which is described in a previous study [4]. Each disk sample was rheologically tested at least 3 times for statistical reasons.

As a synovial fluid substitute, a bovine serum-based fluid exhibiting comparable non-Newtonian behaviour was used. The synovial fluid substitute consists of a foetal bovine serum from the company Capricorn Scientific $\mathrm{GmbH}$ (Ebsdorfergrund, Germany) with a total protein content of 3.7 $\mathrm{g} / \mathrm{dL}$ and hyaluronic acid (HA) with a molecular weight of $2 \times 10^{6}$ Da with a ratio of $3 \mathrm{~g} / \mathrm{L}$. The substitute fluid was applied with the pipette VITLABßmicropipette (VITLAB GmbH, Grossostheim, Germany).
The surface of the disks was investigated in regard to roughness by a laser scanning microscope with a $\lambda_{\mathrm{c}}$ of $80 \mu \mathrm{m}$ (OLS4000, Olympus, Tokyo, Japan). The surface structure in regard to depth, lateral distance and gap width was measured at least three times at a magnification of $10 \mathrm{x}$ and $50 \mathrm{x}$, using a laser scanning microscope (VK-X200, Keyence Corporation, Osaka, Japan). The ring-shaped microstructures consist of a width of $15 \mu \mathrm{m}$ with the following variations of the microstructure dimensions on the disks are given in Table 1 .

Table 1: Specifications of the ring-shaped microstructures

\begin{tabular}{cccccc}
\hline Sample & $\begin{array}{c}\text { Diameter } \\
\text { in } \boldsymbol{\mu} \mathbf{m}\end{array}$ & $\begin{array}{c}\text { Distance } \\
\text { in } \boldsymbol{\mu m}\end{array}$ & $\begin{array}{c}\text { Depth } \\
\text { in } \boldsymbol{\mu m}\end{array}$ & $\begin{array}{c}\text { Aspect } \\
\text { Ratio }\end{array}$ & $\begin{array}{c}\text { Density } \\
\text { in \% }\end{array}$ \\
\hline S1 & 100 & 200 & 20 & 1.33 & 5.9 \\
S2 & 100 & 150 & 20 & 1.33 & 10.5 \\
S3 & 150 & 300 & 20 & 1.33 & 2.5 \\
S4 & 100 & 200 & 10 & 0.66 & 5.9 \\
S5 & 100 & 150 & 10 & 0.66 & 10.5 \\
S6 & 150 & 300 & 10 & 0.66 & 2.5 \\
\hline
\end{tabular}

\section{Results and discussion}

The roughness of the unstructured CoCrMo and $\mathrm{Al} 2 \mathrm{O} 3$ disks was measured at $R_{a}=0.34 \mu \mathrm{m}$ and $R_{a}=0.36 \mu \mathrm{m}$, respectively. Microstructuring of CoCrMo disks was carried out successfully using a femtosecond laser, as seen in Figure 2. The microstructures show a typical laser-generated groove profile (see Figure 3) with high qualities and a minimized burr, resulting in an average width of $\mathrm{a}=14.9 \pm 1.3 \mu \mathrm{m}$ for the CoCrMo specimens and $15.2 \pm 0.9 \mu \mathrm{m}$ for the $\mathrm{Al} 2 \mathrm{O} 3$ specimens, respectively

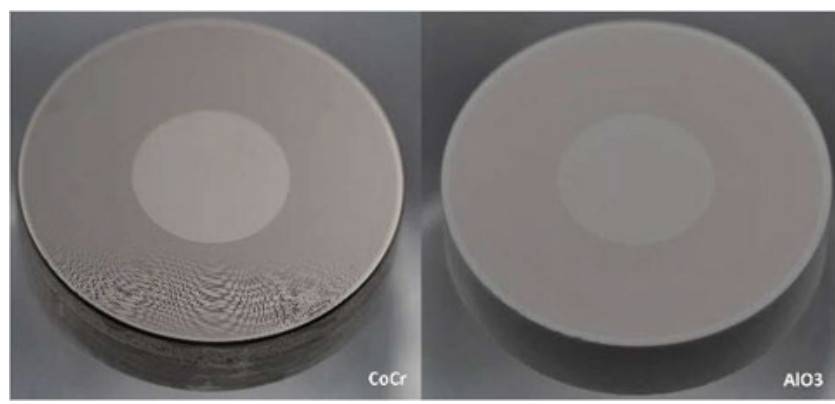

Fig. 2: Microstructured disks (S3) by a femtosecond laser for rheological parallel plate testing (left: CoCrMo, right: Al2O3).

The measurements of the apparent dynamic viscosity $\eta$ ' were carried out at a temperature of $20{ }^{\circ} \mathrm{C}$ and at a constant shear rate of $100 \mathrm{~s}-1$, even though a shear rate dependency of the viscosity of joint fluid is well known. Nonetheless, it was 
necessary to measure the properties in the chosen range in order to effectively compare the samples to each other.

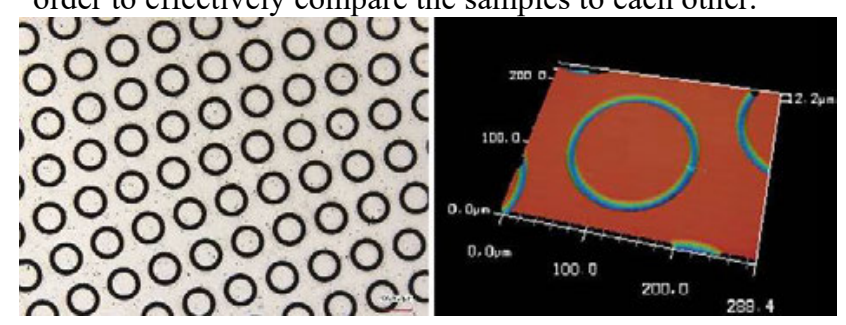

Fig. 3: Exemplary optical analysis of the microstructures of specimen S5: CoCrMo (left: 10x magnification, right: 50x)

As seen in Figure 4 of the rheological measurements, the microstructures have resulted in an overall increase in the apparent dynamic viscosity of the synovial substitute with ring-like structures, in comparison to the reference with an unstructured surface. Differences in the microstructures such as texture density and aspect ratio can be observed to have an impact on the apparent dynamic viscosity, with the CoCrMo sample $\mathrm{S}_{\mathrm{M}}\left(\eta \eta^{\prime}=49.5 \pm 0.3 \mathrm{mPas}\right)$ and the $\mathrm{Al} 2 \mathrm{O} 3$ sample $\mathrm{S} 4_{\mathrm{C}}$ $\left(\eta^{\prime}=61.1 \pm 0.8 \mathrm{mPas}\right)$ showing the largest increase of $30 \%$ and $42 \%$, respectively, compared to the reference sample $(\eta=$ $35.4 \pm 0.7 \mathrm{mPas})$. Furthermore, the specimens with less distance between the ring-shaped microstructures and therefore a higher texture density result in a marginally higher apparent dynamic viscosity with the exception of specimen S6.

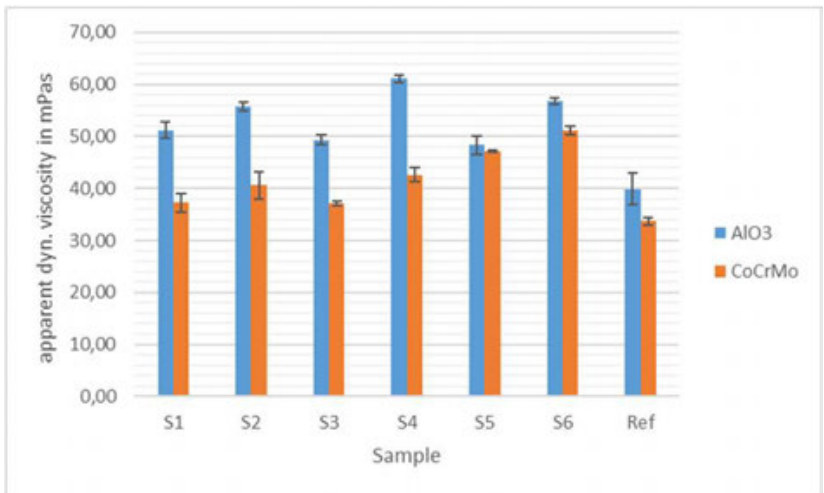

Fig. 4: Results of the parallel plate rheological measurements

This could be due to some combinatory effects between aspect ratio, texture density and microstructure shape, which needs to be investigated further. Looking at the aspect ratio, the samples with 0.66 show a generally higher measurement of the apparent dynamic viscosity, compared to the samples with an aspect ratio of 1.33. However, this trend is not as prevalent in the $\mathrm{A} 12 \mathrm{O} 3$ specimens. It can also be observed that the $\mathrm{Al} 2 \mathrm{O} 3$ specimens reach overall higher dynamic viscosities of approx. $20 \%$ on average. This could be explained by slight variations in the geometric accuracy of the microstructures between ceramic and metallic samples. Additionally, the ceramic specimens exhibit a slightly rougher surface of approx. $7 \%$. The CoCrMo specimens show an average width discrepancy of $2 \%$ and an average depth discrepancy of $5 \%$, compared to the Al2O3 specimens. However, sample S3 shows a slightly higher discrepancy with the depth of a $9 \%$ and width of a $5 \%$ deviation between both materials. This could explain why this specimen is exhibiting a larger deviation than the other specimens.

In regard to the shape of the microstructures, the symmetrical geometry of the ring-shaped structure can be regarded as a positive attribute and could therefore lead to a steadier formation of a lubricant film. The reason for higher apparent dynamic viscosities at microstructured surfaces in comparison to a smooth surface is the local change in gap size which results in a change in the shear rate of the fluid. The microstructures function as wells on surfaces that are a gap enlargement for the flow [13]. The fluid can flow into the wells, lowering shear rates and increase viscosity. Furthermore, these textures can act as wear traps for particles and subsequently improve the life cycle of artificial joints.

\section{Conclusion}

Microstructures were created using a pulsed femtosecond laser with well-defined and sharp contours. The measurement of the apparent dynamic viscosity of a synovia substitute fluid was carried out successfully. The results of the rheological measurements have shown that ring-like microstructures have an increasing effect on the apparent dynamic viscosity of the non-Newtonian test fluid which is hypothesized to improve lubrication and lower the wear rate of the implant resulting in a longer life cycle. The results also show that the influence of different materials is not marginal, and the differences are probably due to the dimensional deviations of the microstructures caused by the laser microstructuring. Further inspection of more microstructures can help to better understand the influence of such parameters and even lead to an optimum value for improved joint lubrication. A possible correlation between the friction coefficient, film thickness, hydrodynamic pressure and viscosity likewise needs to be investigated in future studies to further understand the impact of microstructures on the lubrication of joint prostheses.

\section{Author Statement}

Research funding: This research was funded by the Federal Ministry of Education and Research (funding code: 03WKCU4B; Innovative Regional Growth Cores "MikroLas" which is part of the initiative "Entrepreneurial Regions"- "The 
BMBF Innovation Initiative for the New German Länder"). This work was supported by the German Research Foundation (DFG, grant number INST 264/133-1 FUGB. Conflict of interest: Authors state no conflict of interest. Informed consent: Informed consent has been obtained from all individuals included in this study. Ethical approval: The research related to human use complies with all the relevant national regulations, institutional policies and was performed in accordance with the tenets of the Helsinki Declaration, and has been approved by the authors' institutional review board or equivalent committee.

\section{References}

[1] Borjali, A., Langhorn, J., Monson, K., and Raeymaekers, B. 2017. Using a patterned microtexture to reduce polyethylene wear in metalon-polyethylene prosthetic bearing couples. Wear : an international journal on the science and technology of friction lubrication and wear 392-393, 77-83.

[2] Cho, M. and Choi, H.-J. 2014. Optimization of Surface Texturing for Contact Between Steel and Ultrahigh Molecular Weight Polyethylene Under Boundary Lubrication. Tribol Lett 56, 3, 409-422.

[3] Chyr, A., Qiu, M., Speltz, J., Jacobsen, R. L., Sanders, A. P., and Raeymaekers, B. 2014. A patterned microtexture to reduce friction and increase longevity of prosthetic hip joints. Wear : an international journal on the science and technology of friction lubrication and wear 315, 1-2, 51-57.

[4] Drescher, P., Oldorf, P., Dreier, T., Peters, R., and Seitz, H. 2019. Modification of joint prosthesis surfaces by ultrashort pulse laser treatment for improved joint lubrication. Current Directions in Biomedical Engineering 5, 1, 57-60.

[5] Kung, M., Markantonis, J., Nelson, S., and Campbell, P. 2015. The Synovial Lining and Synovial Fluid
Properties after Joint Arthroplasty. Lubricants 3, 2, 394-412.

[6] Langhorn, J., Borjali, A., Hippensteel, E., Nelson, W., and Raeymaekers, B. 2018. Microtextured CoCrMo alloy for use in metal-on-polyethylene prosthetic joint bearings: multi-directional wear and corrosion measurements. Tribology international 124, 178-183.

[7] Langhorn, J., Borjali, A., Hippensteel, E., Nelson, W., and Raeymaekers, B. 2018. Microtextured CoCrMo alloy for use in metal-on-polyethylene prosthetic joint bearings: multi-directional wear and corrosion measurements. Tribology international 124, 178-183.

[8] M Jagatia and Z M Jin. Elastohydrodynamic lubrication analysis of metal-on-metal hip prostheses under steady state entraining motion.

[9] Myant, C. W. and Cann, P. 2014. The effect of transient conditions on synovial fluid protein aggregation lubrication. Journal of the mechanical behavior of biomedical materials 34, 349-357.

[10] Qiu, M., Chyr, A., Sanders, A. P., and Raeymaekers, B. 2014. Designing prosthetic knee joints with bioinspired bearing surfaces. Tribology international 77, 106-110.

[11] Sawano, H., Warisawa, S.’i., and Ishihara, S. 2009. Study on long life of artificial joints by investigating optimal sliding surface geometry for improvement in wear resistance. Precision Engineering 33, 4, $492-$ 498.

[12] Tarabolsi, M., Klassen, T., Mantwill, F., Gärtner, F., Siegel, F., and Schulz, A.-P. 2013. Patterned CoCrMo and $\mathrm{A} 12 \mathrm{O} 3$ surfaces for reduced free wear debris in artificial joint arthroplasty. Journal of biomedical materials research. Part A 101, 12, 3447-3456.

[13] Tarabolsi, M., Klassen, T., Mantwill, F., Gärtner, F., Siegel, F., and Schulz, A.-P. 2013. Patterned CoCrMo and A12 $\mathrm{O} 3$ surfaces for reduced free wear debris in artificial joint arthroplasty. Journal of biomedical materials research. Part A 101, 12, 3447-3456. 